\title{
Awareness on Topical Therapeutic In Management of Oral Mucosal Lesions Among Undergraduate Dental Students in a University Setting- a Questionnaire Based Survey
}

\author{
Nivethitha. $\mathrm{R}^{1}$ and T. N. Uma Maheswari ${ }^{2}$ \\ ${ }^{1}$ Saveetha Dental College and Hospitals, Saveetha Institute of Medical \\ and technical Sciences, Saveetha University, Chennai, India \\ ${ }^{2}$ Professor and Head of Admin Dept of Oral Medicine and Radiology Saveetha Dental College and \\ Hospitals Saveetha Institute of Medical and Technical Sciences Saveetha University, Chennai , India.
}

\section{ABSTRACT}

Topical medications play an important role in the management of oral lesions. Various topical medications had been extensively used as the first line of therapy in many conditions such as vesiculobullous diseases, oral infections like candidiasis, herpes simplex, potentially malignant disorders and oral mucositis. The aim of this questionnaire study is to assess the awareness of proper treatment protocol of use of topical therapeutics in management of oral mucosal lesion among undergraduate dental students in a university setting. The questionnaire based study was conducted among 100 participants. A self administered questionnaire was used. Sampling was done by convenient sampling. The study was done in a university setting. The questionnaires were distributed to the dental students who have clinical exposure including 3rd BDS, final BDS and interns .It was circulated using an online search software, google form and the response was collected through it. The collected data was ranked as good (11 - 15 correct responses), average ( 6 - 10 correct responses) and below average (below 5 correct responses) then it is tabulated. The data collected were stored and results were analysed by SPSS software. Out of 100, 63\% of the participants were male and 37\% were female. Pearson association was done between the year of study and the awareness percentage, and found to be statistically significant (Chi square value : 60.475 ; $p$-value $=0.00<0.05$ which is statistically significant . Interns showed good awareness compared to the other undergraduate dental students followed by final years who had average awareness on use of topical therapeutic in management of oral mucosal lesions. Most of the third year dental students (29\%) showed below averaged which emphasise a need on educational programs exclusively on topical therapeutics in management of oral mucosal lesions to be initiated from the beginning of clinical exposure for undergraduate students and educational programs on current updates of topical therapeutics is essential for undergraduates to ensure proper treatment protocol in dental practice.

KEY WORDS: TOPICAL THERAPEUTICS ; ORAL MUCOSAL LESIONS ; AWARENESS; UNDERGRADUATE DENTAL STUDENTS.

\section{ARTICLE INFORMATION}

*Corresponding Author: umamaheswaritn@saveetha.com

Received 5th August 2020 Accepted after revision 13th Sep 2020

Print ISSN: 0974-6455 Online ISSN: 2321-4007 CODEN: BBRCBA

Thomson Reuters ISI Web of Science Clarivate Analytics USA and Crossref Indexed Journal

\section{Clarivate
Analytics}




\section{INTRODUCTION}

Oral mucosa is a stratified squamous epithelium, acts as a barrier and protects deeper tissues from trauma, prevents the entry of bacteria and toxic substances entering into the body. Oral mucosa can be the site for both local and systemic drug delivery. Topical application of medication allows the direct action of the drug onto the oral-mucosal lesion, thus increasing its therapeutic effectiveness. Topical drug administration is one among the various routes of drug administration.(Tripathi, 2013)

Topical medications play an important role in the management of oral lesions. Various topical medications ranging from topical anesthetics to topical antineoplastics have been widely used in dentistry. Numerous oral mucosal lesions can be effectively treated by various topical therapeutic agents. Topical medications have been extensively used as the first line of therapy in many conditions such as vesiculobullous diseases, oral infections like candidiasis,oral ulcers, salivary dysfunction, herpes virus simplex, potentially malignant disorders and oral mucositis. Topical drug therapy provides targeted and more efficient drug delivery options for the local oral lesions as compared to systemic therapy. (Hearnden et al., 2012) While accessibility, lower systemic side effects, and many other advantages were associated with the usage of topical oral medications, several challenges are also faced such as taste alterations, poor penetration. Many different formulations such as mucoadhesive tablets, mucoadhesive films, patches, gels, ointments, sprays, and oral rinsed are used for local oral drug delivery.(Hughes and Ghosh, 2015)

Corticosteroids are substances that regulate many functions in our body like fat, protein and carbohydrate metabolism, regulation of the immune system and help to reduce inflammation. In dentistry, corticosteroids are used in a wide variety of conditions such as vesiculobullous ulcerative diseases. They inhibit the inflammatory reaction, redness, and edema. (GonzálezMoles and Scully, 2005) Oral mucosal diseases are common complaints in the outpatient clinic which is caused by a variety of causes that includes local and systemic diseases, trauma, chemical causes,tobacco, and as side effects of certain medications.(Leão, Gomes and Porter, 2007) Oral lesions are usually oral conditions or oral manifestations of systemic diseases. (Field and Allan, 2003)

A study found 28.2\% of American adults to have a diagnosed oral lesion with different prevalence rates. Candida mucosal lesions represented 8.7\%, particularly among denture wearers and the traumatic lesions accounted for 5.7\%, while herpes labialis and herpes gingivostomatitis was found among $1.6 \%$ of the adult population. (Parashar, 2011) The prevalence of oral lichen planus among adults is varied from $0.5 \%$ to $2 \%$ 4,5 , while oral submucous fibrosis is uncommon disease mainly affecting the population in southeastern Asia with a prevalence of 16 per 100,000 (Eisen et al., 2005) In Saudi Arabia, the prevalence of aphthous ulcers among females was 9.8\% while 11\% of Saudi females had lichen planus .(Yang et al., 2018)(Ajmal et al., 2018)

Dental students should be aware about the management of oral mucosal diseases as well as the major oral diseases such as dental caries, gingivitis and periodontitis. The knowledge of dental students is expected to increase by the increase in educational level, and the dental practices are usually improved in the internship year. The aim of this questionnaire study is to assess the awareness of proper treatment protocol of use of topical therapeutics in management of oral mucosal lesion among undergraduate dental students in a university setting. Our recent research portfolio slides numerous articles in reputed journals (Santosh R. Patil et al., 2018; S. R. Patil et al., 2018; Subramaniam and Muthukrishnan, 2019; Vadivel et al., 2019; Patil et al., 2020). Based on this experience we planned to pursue the awareness of proper treatment protocol of use of topical therapeutics in management of oral mucosal lesion among undergraduate dental students in a university setting.

\section{MATERIAL AND METHODS}

The setting was an online University setting. Ethical approval was obtained from the institution's ethical committee and the ethical approval was SRB/SDC/ UG-036/03. The number of people involved in this study includes a guide, two reviewers and one principal investigator. A structured self assessed online questionnaire having 15 questions on topical therapeutics for oral mucosal lesion was prepared with the aim to assess the awareness of proper treatment protocol of use of topical therapeutics in management of oral mucosal lesion among undergraduate dental students in a university setting. Sampling was done by convenient sampling.

The study was done in a university setting. Inclusion criteria of the study includes dental undergraduates students with clinical exposure such as 3rd BDS , final BDS, Interns. Exclusion criteria includes dental undergraduates students without clinical exposure such as 1 st BDS and 2nd BDS. The questionnaire was validated with two reviewers who were experts in the field of oral medicine before distributing to the undergraduate dental students. The questionnaires were distributed to the dental students who have clinical exposure including 3rd BDS, final BDS and interns . It was circulated using an online search software, google form and the response was collected through it.

The collected data was ranked as good (11 - 15 correct responses), average (6 - 10 correct responses) and below average (below 5 correct responses) then it is tabulated. The Collected data was recorded in the Microsoft Excel 2016 and later exported to the Statistical package of Social Science for Windows Statistical analysis was done using SPSS software. (Version 20.0, SPSS Inc., Chicago, Illinois, USA). Frequency distribution of each response among undergraduate dental students such 3rd BDS, final BDS and interns was analysed. Chi-square association 
using Pearson correlation was done to determine the statistical significant association between year of study and the responses for each question. Pearson correlation was done to determine the statistical significant association was done between the year of study and the awareness of topical therapeutics in management of oral mucosal lesions.

\section{RESULTS AND DISCUSSION}

This study was conducted among 100 dental students. Out of 100, 63\% of the participants were male (blue) and 37\% were female (green) [Graph 1]. Pie chart represents 39\% of the participants were 3rd years (blue), 25\% were 4th years (green), 36\% were interns (beige) [Graph 2]. Pie chart represents 50\% of the participants reported aphthous ulcer (green), 28\% were traumatic ulcer (blue), $22 \%$ were herpetic ulcer (beige) [Graph 3]. Pie chart represents $40 \%$ of the participants reported topical steroids (beige), 39\% topical antiseptic (blue), 21\% topical anesthetics (green) [Graph 4]. Pie chart represents 43\% of the participants reported triamcinolone acetonide orobase $0.1 \%$ (blue), 39\% amlexanox paste 5\% (beige), 18\% chlorhexidine gluconate gel $0.1 \%$ ( green)[Graph 5].

Graph 1: Pie chart showing the frequency distribution of year of study. 39\% of the participants were 3rd years (blue), 25\% were 4th years (green), 36\% were interns (beige).

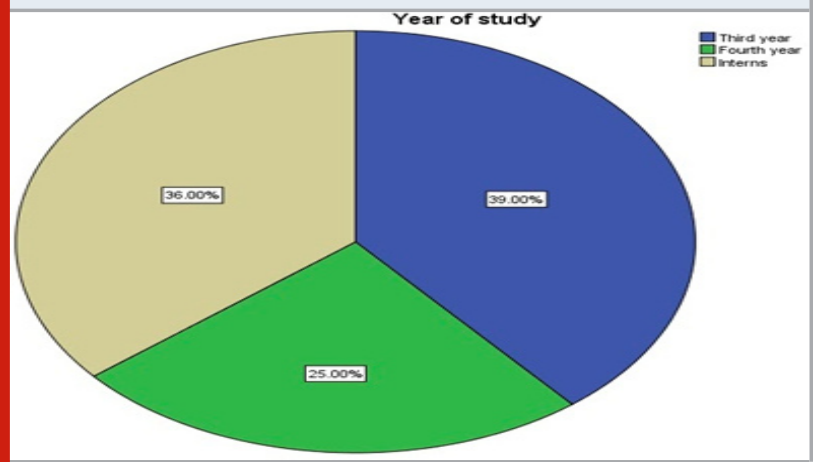

Graph 2: Pie chart showing the frequency distribution of gender. $63 \%$ of the participants were male and $37 \%$ were female

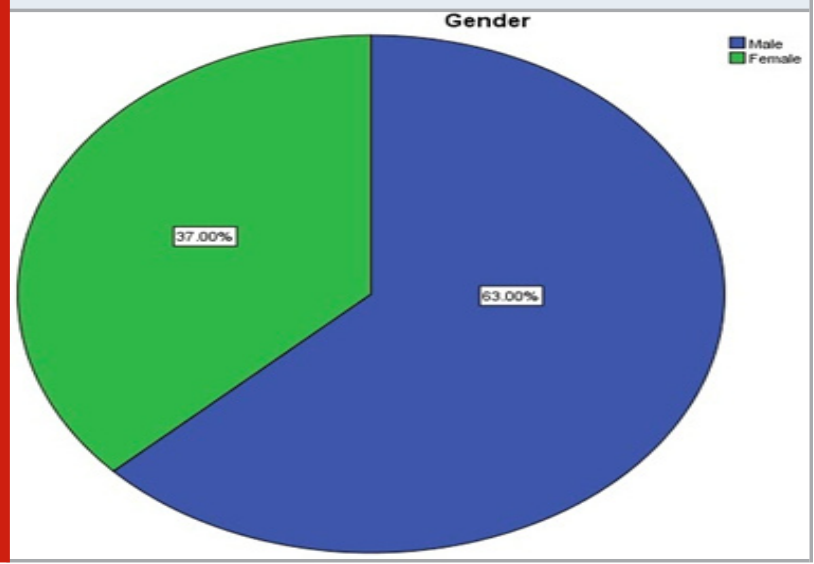

Graph 3: Pie chart showing the responses to the question: "Most common oral mucosal ulcers encountered in your practice for which topical therapeutics is mainly used"? $50 \%$ of the participants were reported aphthous ulcer (green), 28\% were traumatic ulcer (blue), 22\% were herpetic ulcer (beige).

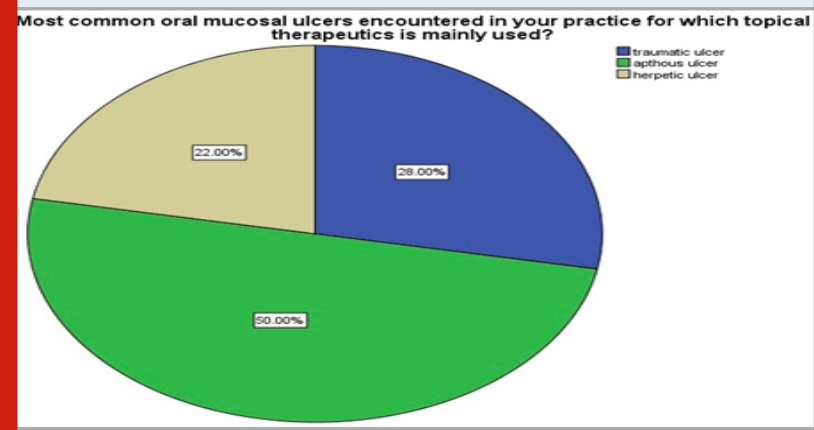

Graph 4: Pie chart showing the responses to the question: "What is the commonly used topical therapeutic for the treatment of traumatic ulcer"? 40\% of the participants reported topical steroids (beige), 39\% topical antiseptic (blue), 21\% topical anesthetics (green).

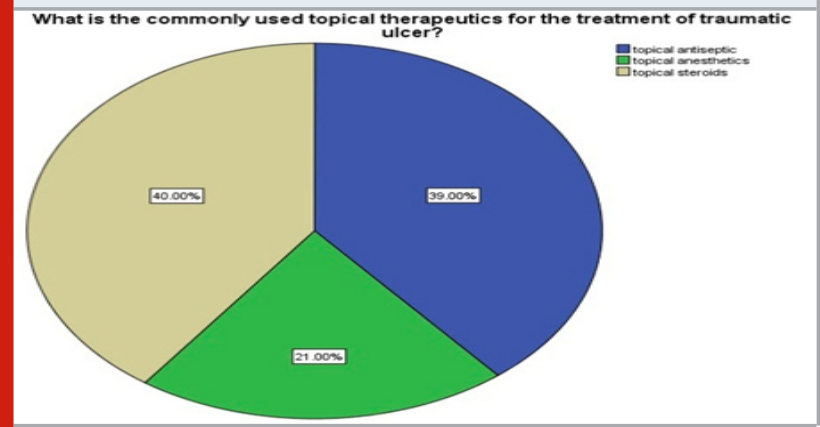

Graph 5: Pie chart showing the responses to the question:

"What is the commonly used topical therapeutic in management of aphthous ulcer"? 43\% of the participants reported triamcinolone acetonide oro-base $0.1 \%$ (blue), $39 \%$ amlexanox paste 5\% (beige), 18\% chlorhexidine gluconate gel $0.1 \%$ ( green).

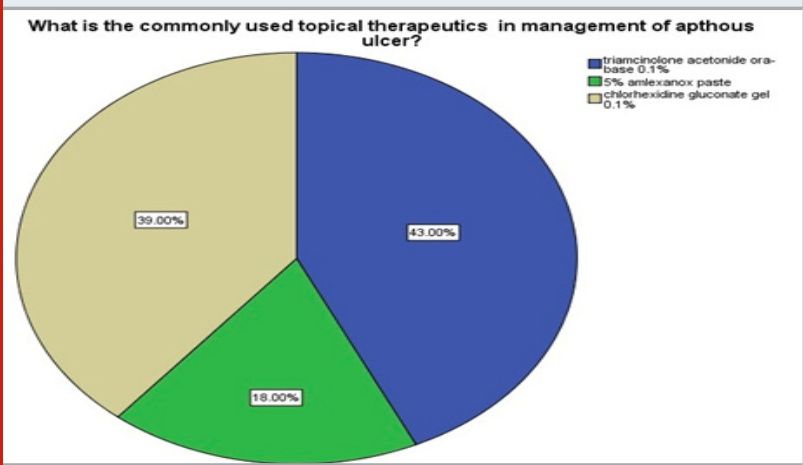

Pie chart represents $43 \%$ of the participants reported acyclovir cream 5\% (beige), 19\% benzocaine I.P 20\% (green), 38\% chlorhexidine gluconate gel 0.1\% (blue) [Graph 6]. Pie chart represents 46\% of the participants reported triamcinolone acetonide oro-base $0.1 \%$ 
(blue), 29\% clobetasol ointment 0.05\% (green), 25\% betamethasone cream $0.1 \%$ (beige) [Graph 7]. Pie chart represents $49 \%$ of the participants reported clotrimazole mouth paint 1\% (beige), 30\% nystatin cream (blue), 21\% ketoconazole (green) [Graph 8]. Pie chart represents 42\% of the participants reported triamcinolone acetonide orobase $0.1 \%$ (beige), 35\% diphenhydramine hydrochloride (blue), 23\% dicyclomine hydrochloride (green)[ Graph 9]. Pie chart represents $41 \%$ of the participants reported hydroxy methyl cellulose (blue), 40\% cevimeline (green) , 19\% natural sialogogues (beige) [Graph 10].

Graph 6: Pie chart showing the responses to the question: "What topical therapeutics recommended by you in your practice for herpetic ulcer"? 43\% of the participants reported acyclovir cream 5\% (beige), 19\% benzocaine I.P 20\% (green), 38\% chlorhexidine gluconate gel $0.1 \%$ (blue).

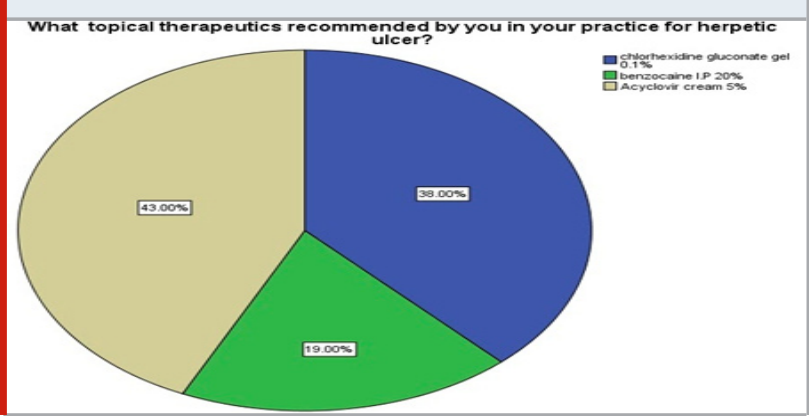

Graph 7: Pie chart showing the responses to the question: "What topical steroids usually prescribed by you in your dental practice"? $46 \%$ of the participants reported triamcinolone acetonide oro-base $0.1 \%$ (blue), 29\% clobetasol ointment $0.05 \%$ (green), $25 \%$ betamethasone cream $0.1 \%$ (beige).

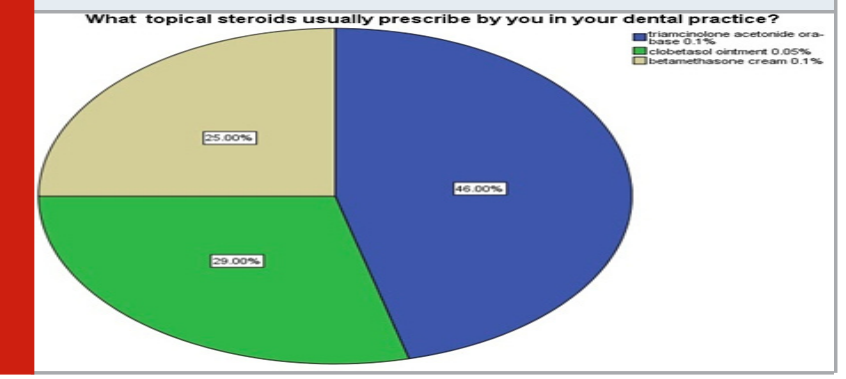

Graph 8: Pie chart showing the responses to the question: "What topical therapeutic used for the treatment of oral candidiasis"? 49\% of the participants reported clotrimazole mouth paint 1\% (beige), 30\% nystatin cream (blue), 21\% ketoconazole (green).

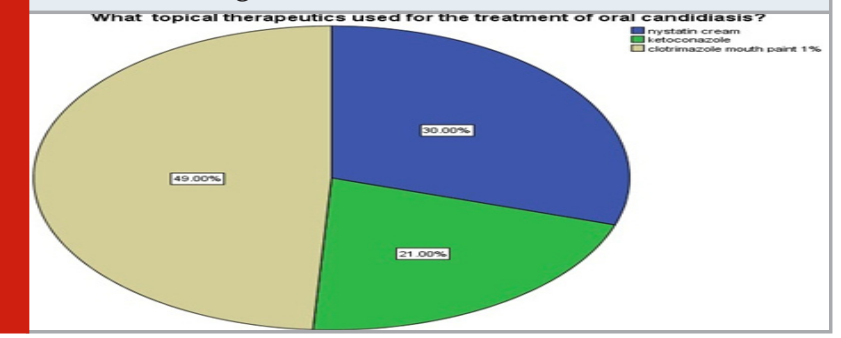

Graph 9: Pie chart showing the responses to the question: "Drug of choice for allergic stomatitis"? 42\% of the participants reported triamcinolone acetonide oro-base $0.1 \%$ (beige), 35\% diphenhydramine hydrochloride (blue), 23\% dicyclomine hydrochloride (green).

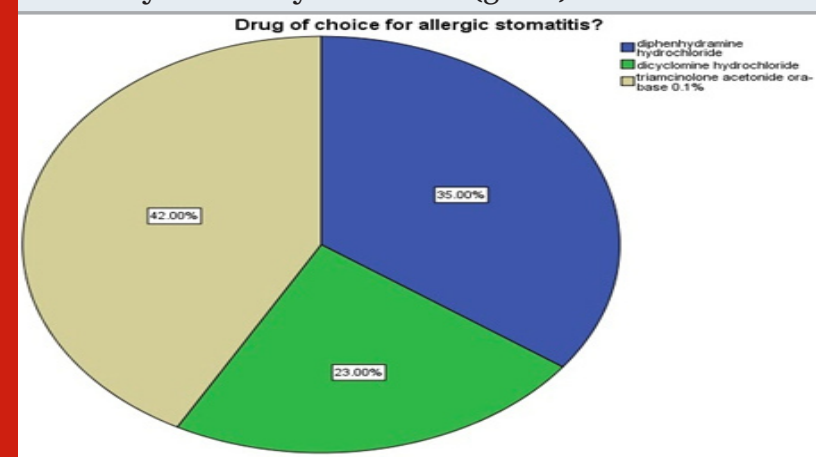

Graph 10: Pie chart showing the responses to the question: "Topical therapeutic in management of xerostomia"? 41\% of the participants reported hydroxy methyl cellulose (blue), 40\% cevimeline (green) , 19\% natural sialogogues (beige).

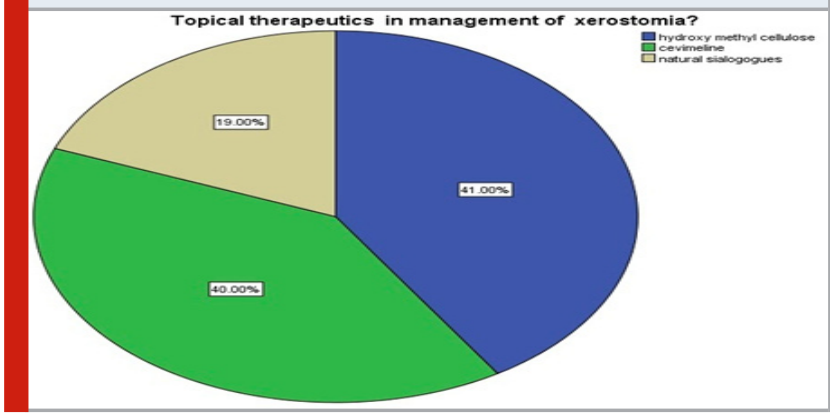

Graph 11: Pie chart showing the responses to the question: "Which is the most common formulation in which topical steroids is administered in oral mucosal lesions"? 53\% of the participants reported orabase (beige), 28\% gel (green) , 19\% Ointment (blue).

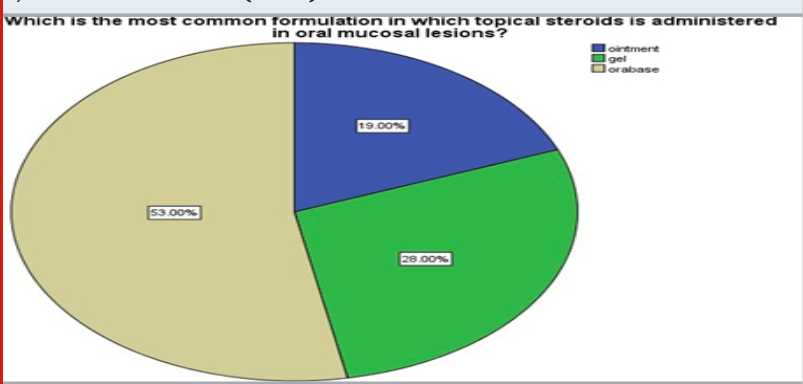

Pie chart represents 53\% of the participants reported orabase (beige), 28\% gel (green), 19\% Ointment (blue) [Graph 11]. Pie chart represents 44\% of the participants reported mucosal atrophy (blue), 36\% candidiasis (green), 20\% exaggeration of lesions on withdrawal (beige) [Graph 12]. Pie chart represents 56\% of the participants reported oral lichen planus (green), 24\% allergic stomatitis (beige), 20\% candidiasis (blue) [Graph 13]. Pie chart represents $44 \%$ of the participants reported 
Triamcinolone acetate 0.1\% (beige), 29\% fluocinonide 0.1\% (blue) , 27\% hydrocortisone butyrate 0.1\% (green) [Graph 14]. Pie chart represents 56\% of the participants reported hexidine mouth rinse (beige), 25\% tantrum mouthrinse (green), 19\% betadine mouth rinse (blue) [Graph 15]. Pie chart represents $44 \%$ of the participants reported hyaluronidase (blue), 34\% dexamethasone and hyaluronidase (beige), 22\% placentrex (green) [Graph 16]. Pie chart represents $41 \%$ of the participants reported lycopene (blue), 40\% retinoids (green), 19\% beta carotene (beige) [Graph 17].

Graph 12: Pie chart showing the responses to the question: "Awareness on side effects of oral topical steroids "? $44 \%$ of the participants reported mucosal atrophy (blue), $36 \%$ candidiasis (green) , 20\% exaggeration of lesions on withdrawal (beige).

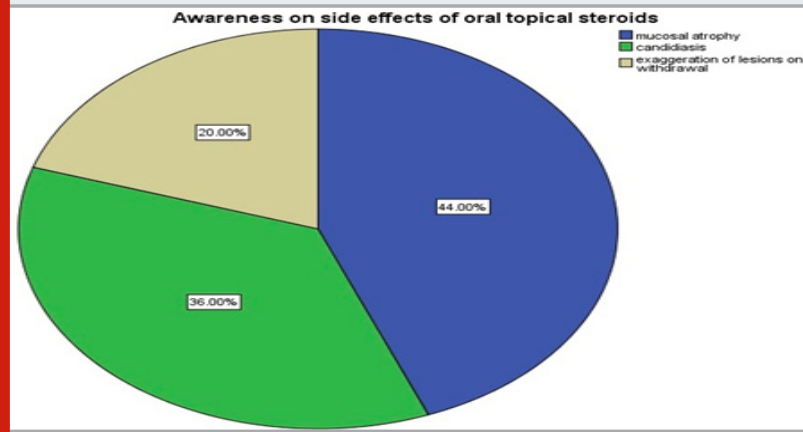

Graph 13: Pie chart showing the responses to the question: "Which is the most common oral mucosal lesion for which you prescribe topical steroids"? 56\% of the participants reported oral lichen planus (green), 24\% allergic stomatitis (beige) , 20\% candidiasis (blue).

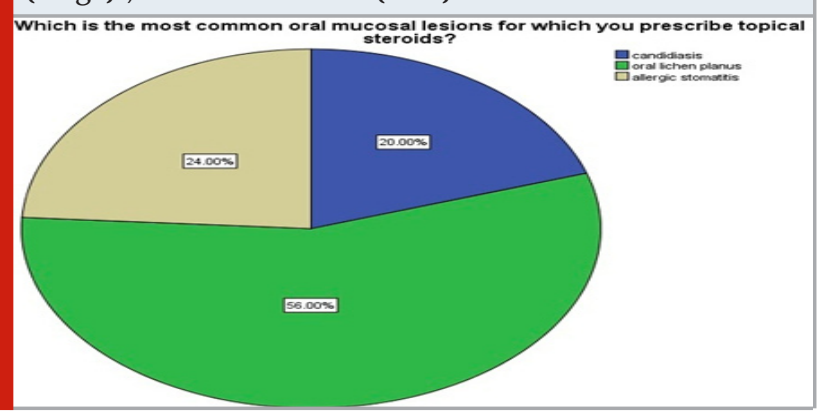

Graph 14: Pie chart showing the responses to the question: "Which one is the most common topical steroids in management of oral lichen planus"? 44\% of the participants reported Triamcinolone acetate $0.1 \%$ (beige), 29\% fluocinonide $0.1 \%$ (blue) , 27\% hydrocorticosone butyrate $0.1 \%$ (green).

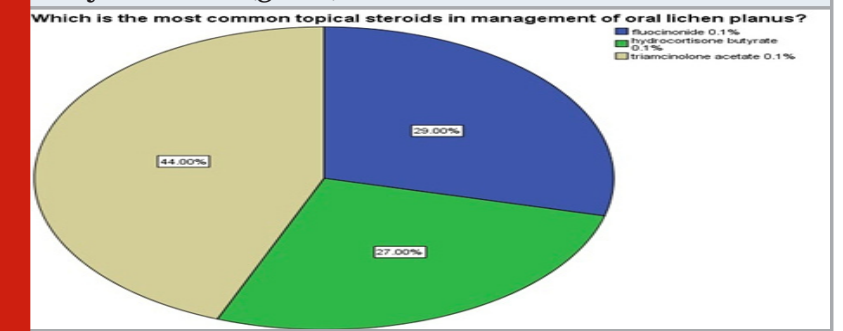

Graph 15: Pie chart showing the responses to the question: "Most common antiseptic mouth rinse prescribed by you in your dental practice"? 56\% of the participants reported hexidine mouth rinse (beige), 25\% tantrum mouthrinse (green) , 19\% betadine mouth rinse (blue).

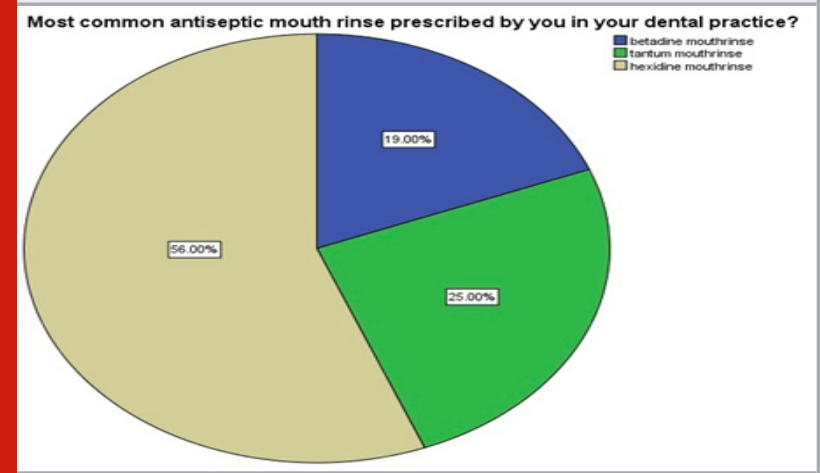

Graph 16: Pie chart showing the responses to the question: "Which of the following is used in the management of OSMF"? 44\% of the participants reported hyaluronidase (blue), 34\% dexamethasone and hyaluronidase (beige) , $22 \%$ placentrex (green).

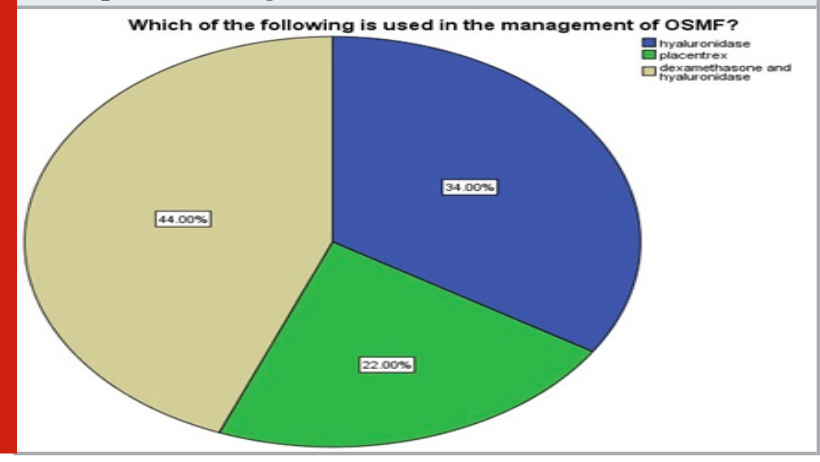

Graph 17: Pie chart showing the responses to the question: "Topical therapeutic used in treatment of oral leukoplakia?" $41 \%$ of the participants reported lycopene (blue), 40\% retinoids (green), 19\% beta carotene (beige).

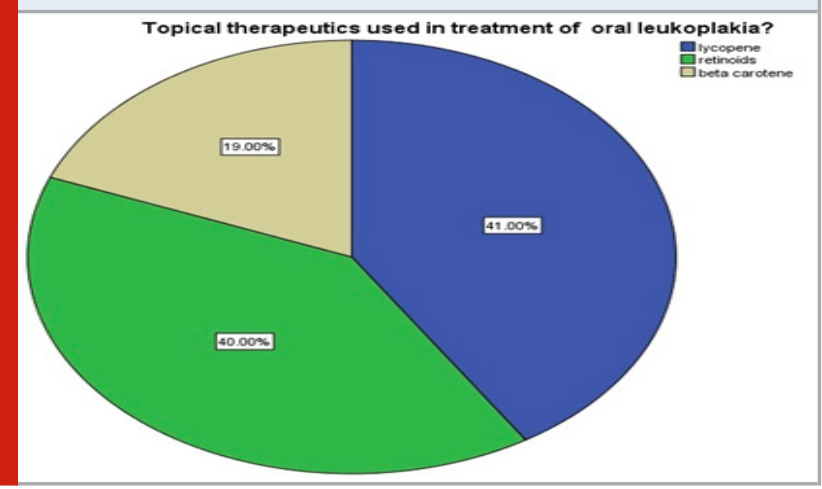

Majority of respondents reported that aphthous ulcers were more common ulcers encountered in their practice for which topical therapeutics is mainly used (Pearson chi square;p -value $=0.00<0.05$ which is statistically significant )[Graph 18]. Majority of respondents reported 
that topical antiseptics were commonly used topical therapeutic for the treatment of traumatic ulcers .(Pearson chi square $; p$-value $=0.00<0.05$ which is statistically significant )[Graph 19]. Majority of respondents reported that triamcinolone acetonide ora-base $0.1 \%$ were commonly used topical therapeutic in management of aphthous ulcer. (Pearson chi square ; $p$-value $=0.049$ $<0.05$ which is statistically significant )[Graph 20]. Majority of respondents reported that acyclovir cream were the topical therapeutics recommended by you in your practice for herpetic ulcer. (Pearson chi square ; $p$ -value $=0.00<0.05$ which is statistically significant ) [Graph 21].

Graph 18: Bar graph showing comparison of responses based on year of study to the question, " Most common oral mucosal ulcers encountered in your practice for which topical therapeutics is mainly used?" $\mathrm{X}$ axis represents the questions and $\mathrm{Y}$ axis represents the number of participants .Majority of respondents reported that aphthous ulcer were more common ulcer encountered in their practice for which topical therapeutics is mainly used(Pearson chi square value- $24.972 ; p$-value $=0.00<0.05$ which is statistically significant ).

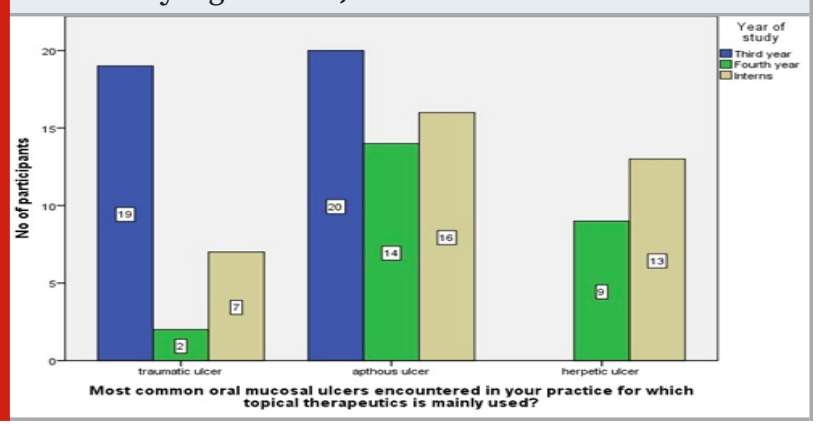

Graph 19: Bar graph showing comparison of responses based on year of study to the question, "What is the commonly used topical therapeutic for the treatment of traumatic ulcer?" $\mathrm{X}$ axis represents the questions and $\mathrm{Y}$ axis represents the number of participants. Majority of respondents reported that topical antiseptics were commonly used topical therapeutic for the treatment of traumatic ulcer .(Pearson chi square value - $26.969 ; \mathrm{p}$ -value $=0.00<0.05$ which is statistically significant ).

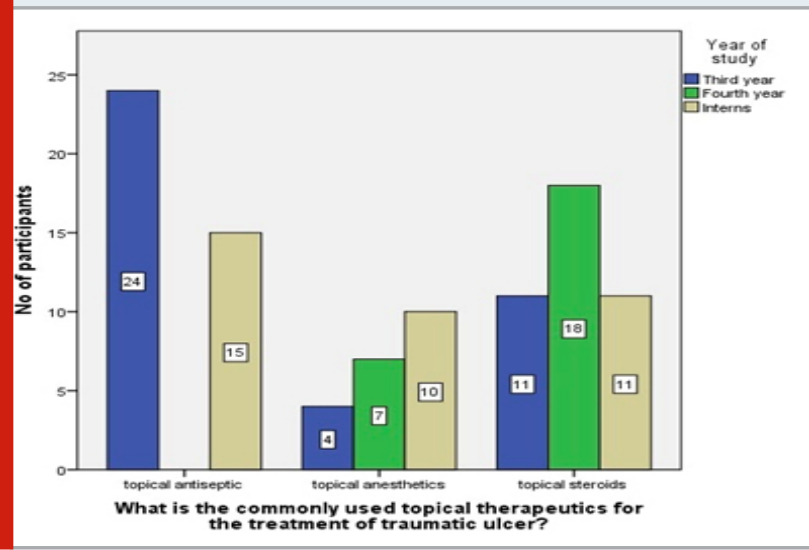

Graph 20: Bar graph showing comparison of responses based on year of study to the question, "What is the commonly used topical therapeutic in management of aphthous ulcer?" $\mathrm{X}$ axis represents the questions and $\mathrm{Y}$ axis represents the number of participants. Majority of respondents reported that triamcinolone acetonide orabase $0.1 \%$ were commonly used topical therapeutic in management of aphthous ulcer. (Pearson chi square value - $36.567 ; p$-value $=0.00<0.05$ which is statistically significant ).

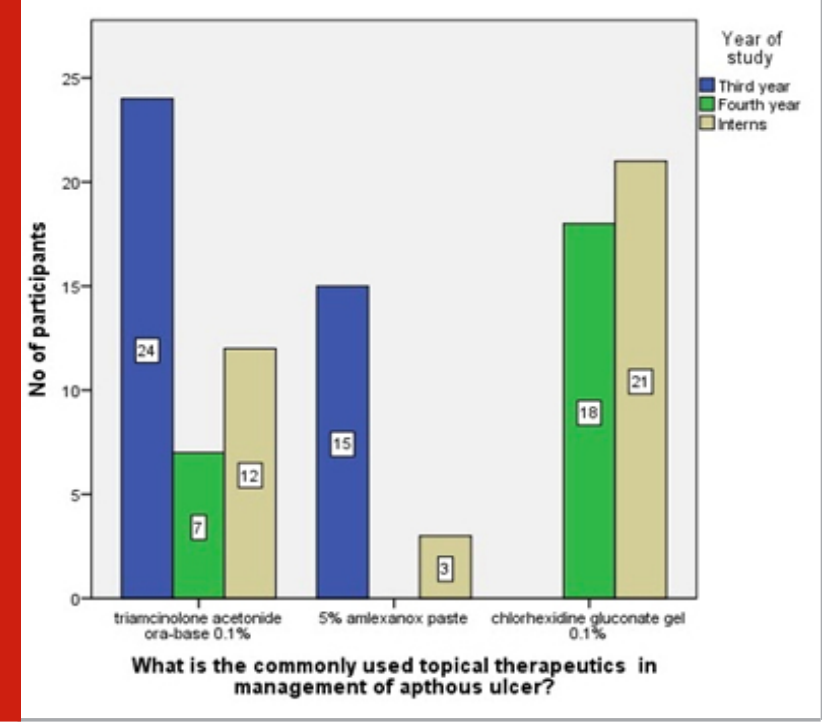

Graph 21: Bar graph showing comparison of responses based on year of study to the question, " What topical therapeutics recommended by you in your practice for herpetic ulcer?" $\mathrm{X}$ axis represents the questions and $\mathrm{Y}$ axis represents the number of participants .Majority of respondents reported that acyclovir cream were the topical therapeutics recommended by you in your practice for herpetic ulcer. (Pearson chi square value - 9.547 ; p -value $=0.049<0.05$ which is statistically significant ).

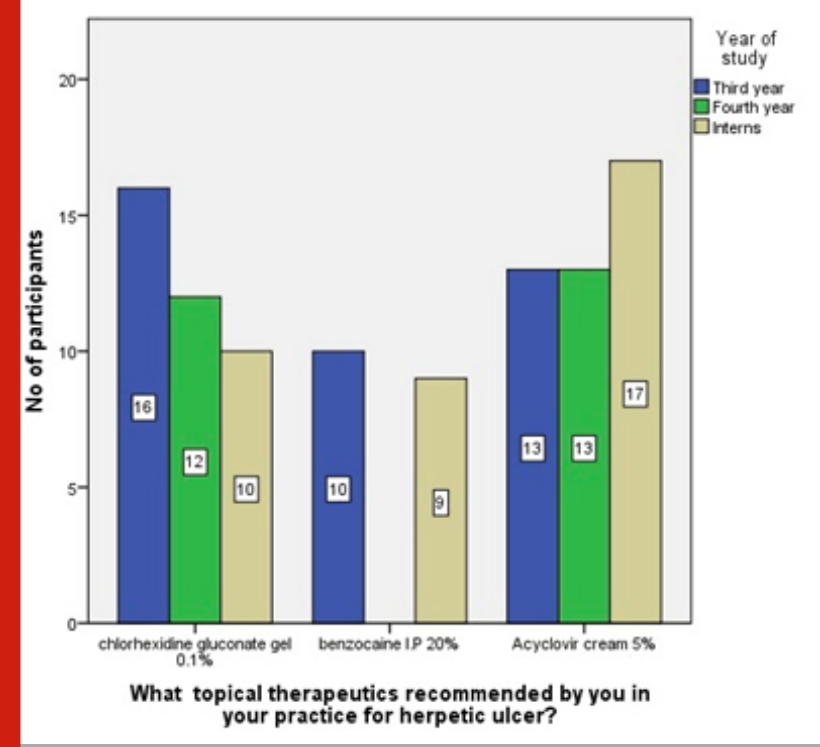


Graph 22: Bar graph showing comparison of responses based on year of study to the question, "What topical steroids usually prescribed by you in your dental practice?" $\mathrm{X}$ axis represents the questions and $\mathrm{Y}$ axis represents the number of participants. Majority of respondents reported that triamcinolone acetonide Orabase $0.1 \%$ were the topical steroids usually prescribed by them in their dental practice(Pearson chi square value - 8.557 ; $\mathrm{p}$-value $=$ $0.073>0.05$ which is statistically not significant ).

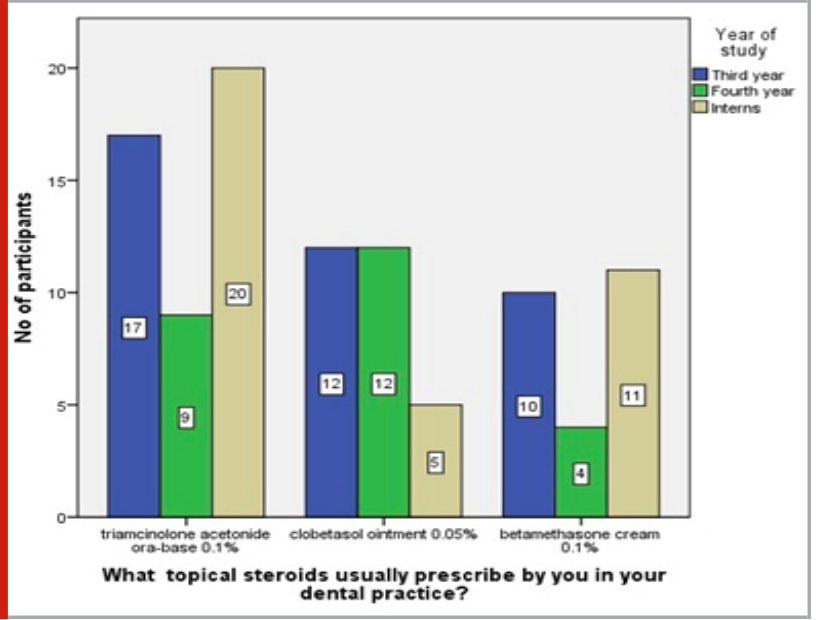

Graph 23: Bar graph showing comparison of responses based on year of study to the question, " Drug of choice for allergic stomatitis?” X axis represents the questions and $\mathrm{Y}$ axis represents the number of participants .Majority of respondents reported that triamcinolone acetonide orabase $0.1 \%$ were the drug of choice for allergic stomatitis. (Pearson chi square value - $37.934 ;$ p -value $=0.00<$ 0.05 which is statistically significant ).

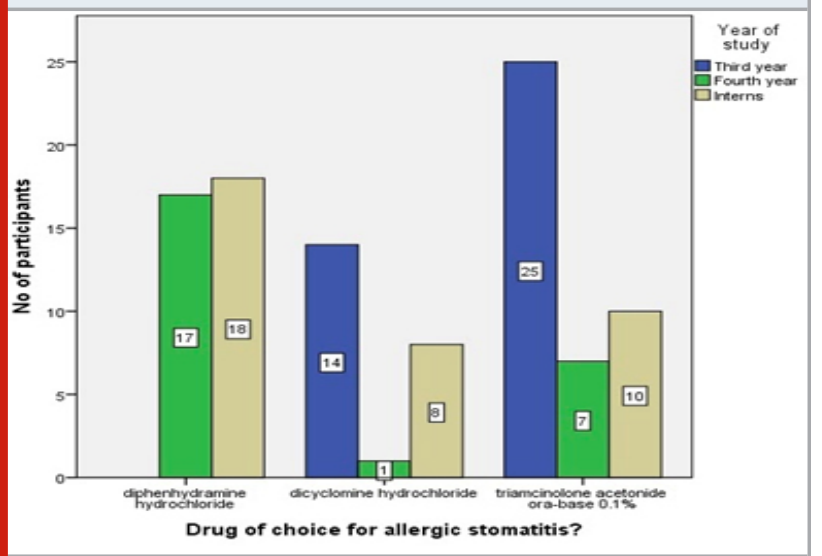

Majority of respondents reported that triamcinolone acetonide Orabase $0.1 \%$ were the topical steroids usually prescribed by them in their dental practice(Pearson chi square $; p$-value $=0.073>0.05$ which is statistically not significant )[Graph 22]. Majority of respondents reported that triamcinolone acetonide orabase $0.1 \%$ were the drug of choice for allergic stomatitis.(Pearson chi square ; p -value $=0.00<0.05$ which is statistically significant )[Graph 23]. Majority of respondents reported that cevimeline was the topical therapeutic in management of xerostomia. (Pearson chi square ; p -value $=0.00<0.05$ which is statistically significant ) [Graph 24]. Majority of respondents reported that retinoids were the topical therapeutic used in treatment of oral leukoplakia (Pearson chi square $; p$-value $=0.00<0.05$ which is statistically significant )[Graph 25].

Graph 24: Bar graph showing comparison of responses based on year of study to the question, " Topical therapeutic in management of xerostomia?" $X$ axis represents the questions and $\mathrm{Y}$ axis represents the number of participants. Majority of respondents reported that cevimeline was the topical therapeutic in management of xerostomia. (Pearson chi square value - $35.118 ; p$-value $=0.00<0.05$ which is statistically significant).

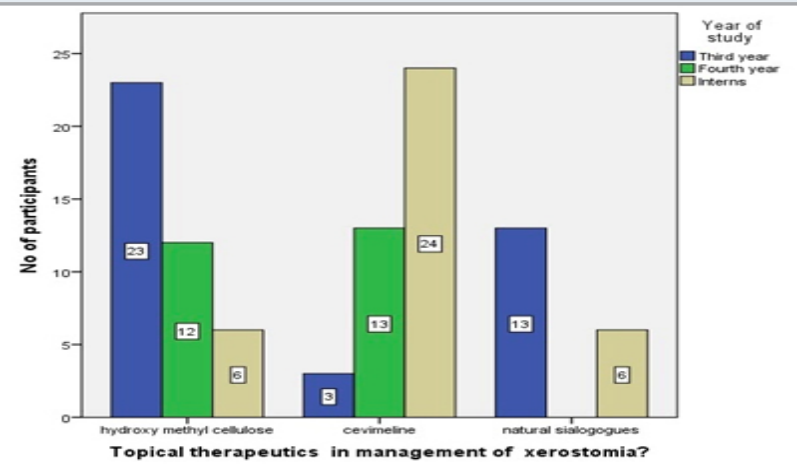

Graph 25: Bar graph showing comparison of responses based on year of study to the question, " Topical therapeutic used in treatment of oral leukoplakia?” X axis represents the questions and $\mathrm{Y}$ axis represents the number of participants Majority of respondents reported that retinoids were the topical therapeutic used in treatment of oral leukoplakia (Pearson chi square value - 35.118 ; $p$ -value $=0.00<0.05$ which is statistically significant ).

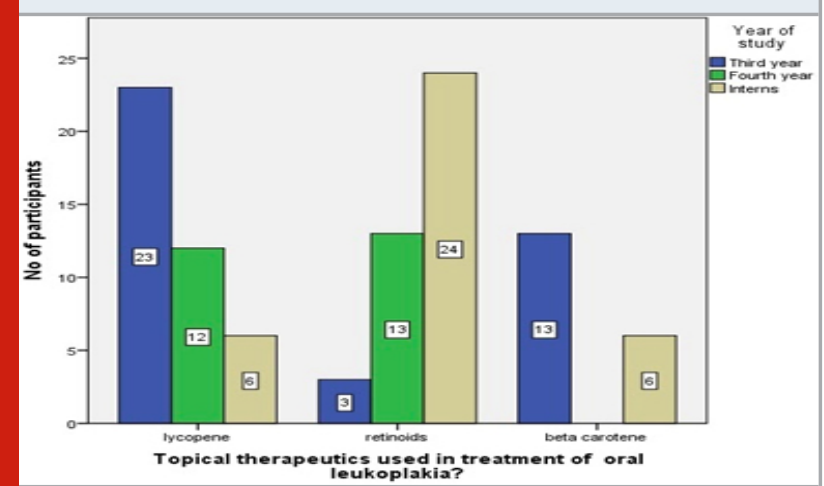

Majority of respondents reported that ora base was the most common formulation in which topical steroids are administered in oral mucosal lesions (Pearson chi square $; \mathrm{p}$-value $=0.001<0.05$ which is statistically significant )[Graph 26]. Majority of respondents reported that mucosal therapy were the side effects of oral topical steroids.(Pearson chi square ; p -value $=0.00<0.05$ which is statistically significant) [Graph 27].Majority of respondents reported that oral lichen planus was the most common oral mucosal lesion for which they 
prescribe topical steroids (Pearson chi square ; $p$-value $=0.00<0.05$ which is statistically significant )[Graph 28]. Majority of respondents reported that triamcinolone acetate $0.1 \%$ were the common topical steroids in management of oral lichen planus.(Pearson chi square ; $\mathrm{p}$-value $=0.00<0.05$ which is statistically significant )[Graph 29].

Graph 26: Bar graph showing comparison of responses based on year of study to the question, "Which is the most common formulation in which topical steroids is administered in oral mucosal lesions?" $\mathrm{X}$ axis represents the questions and $\mathrm{Y}$ axis represents the number of participants .Majority of respondents reported that ora base was the most common formulation in which topical steroids is administered in oral mucosal lesions (Pearson chi square value - $19.741 ; p$-value $=0.001<0.05$ which is statistically significant).

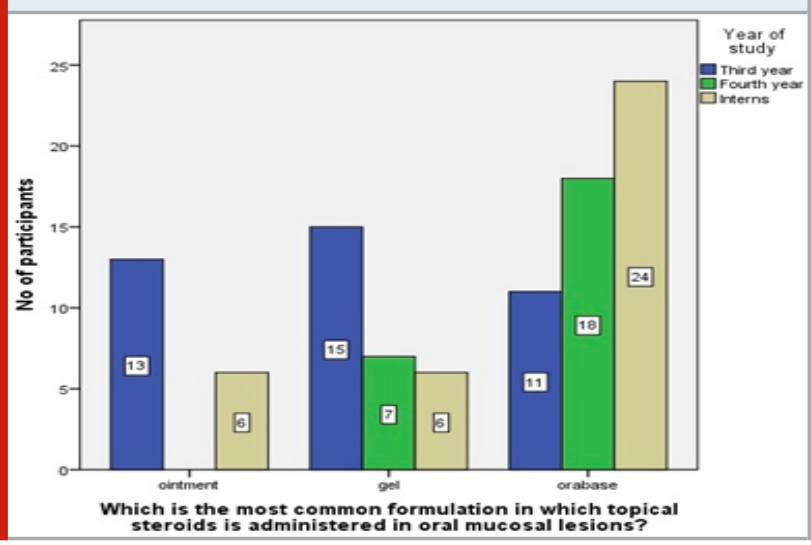

Graph 27: Bar graph showing comparison of responses based on year of study to the question, "Awareness on side effects of oral topical steroids?" $\mathrm{X}$ axis represents the questions and $\mathrm{Y}$ axis represents the number of participants . Majority of respondents reported that mucosal therapy were the side effects of oral topical steroids.(Pearson chi square value - $66.686 ; p$-value $=0.00<0.05$ which is statistically significant ).

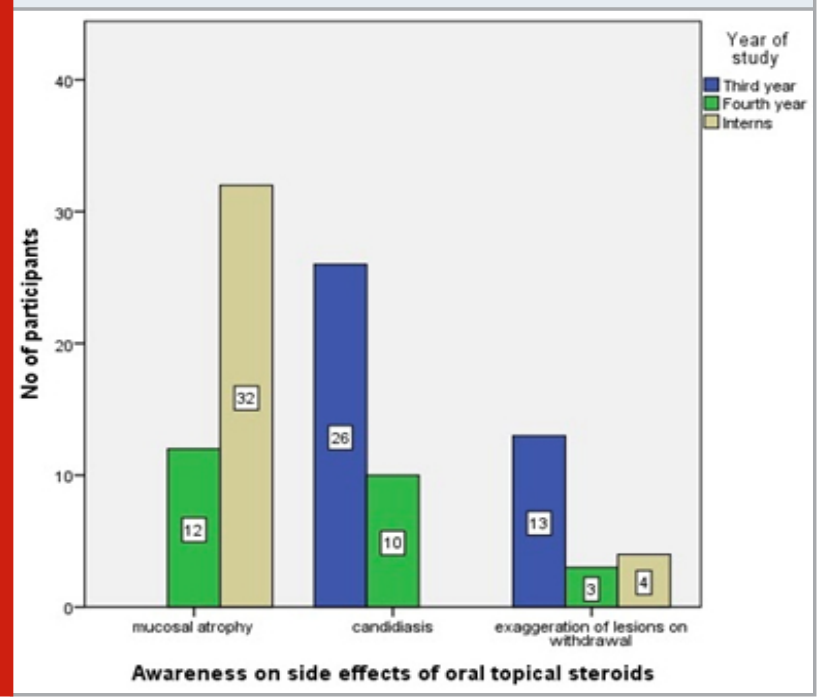

Graph 28: Bar graph showing comparison of responses based on year of study to the question, "Which is the most common oral mucosal lesion for which you prescribe topical steroids?" $\mathrm{X}$ axis represents the questions and $\mathrm{Y}$ axis represents the number of participants .Majority of respondents reported that oral lichen planus was the most common oral mucosal lesion for which they prescribe topical steroids (Pearson chi square value - 71.270 ; $p$ -value $=0.00<0.05$ which is statistically significant).

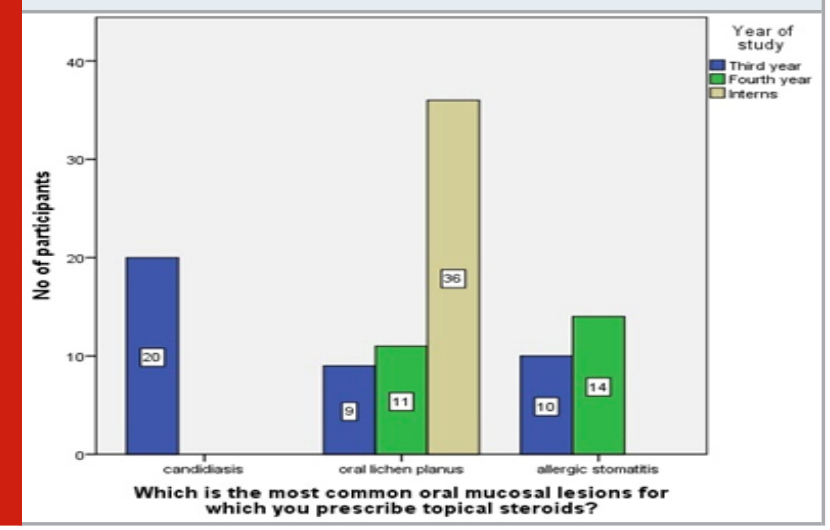

Graph 29: Bar graph showing comparison of responses based on year of study to the question, "Which one is the most common topical steroids in management of oral lichen planus?" $\mathrm{X}$ axis represents the questions and $\mathrm{Y}$ axis represents the number of participants .Majority of respondents reported that triamcinolone acetate $0.1 \%$ were the common topical steroids in management of oral lichen planus.(Pearson chi square value - $50.967 ; \mathrm{p}$-value = $0.00<0.05$ which is statistically significant).

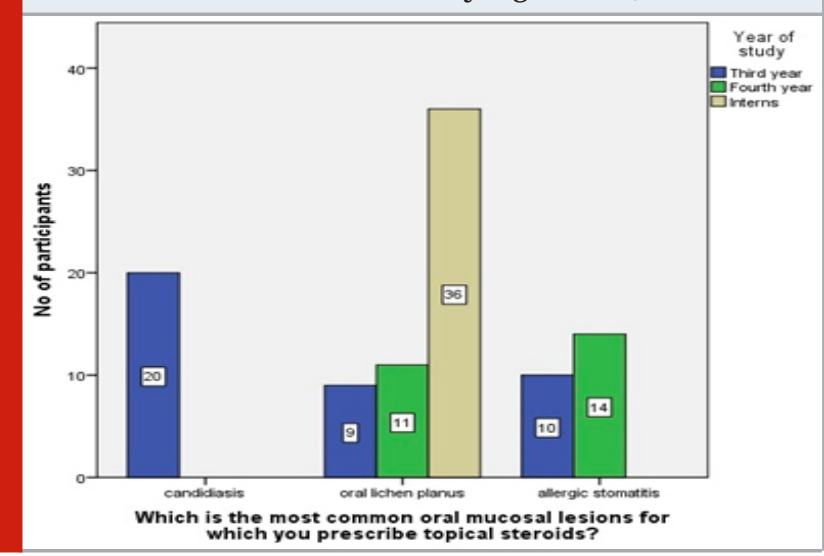

Majority of respondents reported that hyaluronidase was used in the management of OSMF. (Pearson chi square ; $\mathrm{p}$-value $=0.00<0.05$ which is statistically significant ) [Graph 30]. Majority of respondents reported that clotrimazole mouth paint $1 \%$ were topical therapeutic is used for the treatment of oral candidiasis. (Pearson chi square $; p$-value $=0.00<0.05$ which is statistically significant )[Graph 31]. Majority of respondents reported that hexidine mouth rinse were the most common antiseptic mouth rinse prescribed by them in their dental practice(Pearson chi square $; \mathrm{p}$-value $=0.00<0.05$ which 
is statistically significant )[Graph 32].Interns were more aware about topical therapeutics in management of oral mucosal lesions. (Pearson chi square ; p -value $=0.00<$ 0.05 which is statistically significant ) [Graph 33].

Graph 30: Bar graph showing comparison of responses based on year of study to the question, "Which of the following is used in the management of OSMF" ?X axis represents the questions and $\mathrm{Y}$ axis represents the number of participants. Majority of respondents reported that hyaluronidase was used in the management of OSMF. (Pearson chi square value - 64.590; $\mathrm{p}$-value $=0.00<$ 0.05 which is statistically significant).

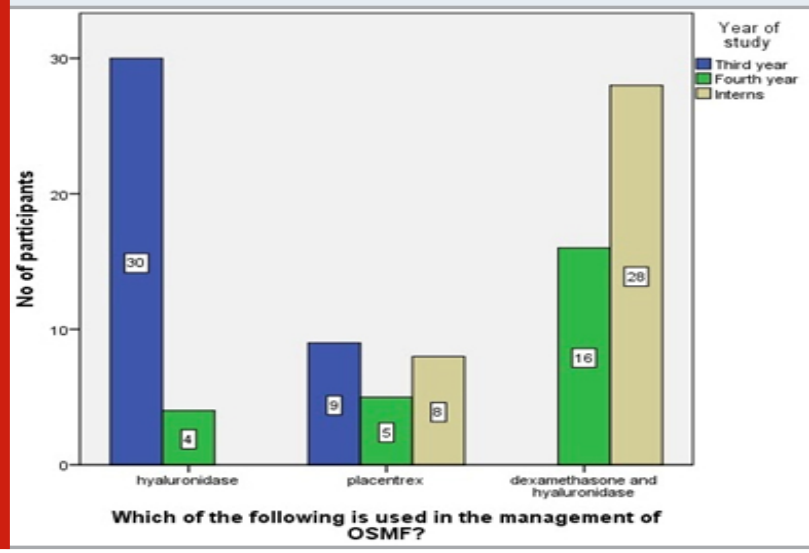

Graph 31: Bar graph showing comparison of responses based on year of study to the question, "What topical therapeutic is used for the treatment of oral candidiasis?" $\mathrm{X}$ axis represents the questions and $\mathrm{Y}$ axis represents the number of participants .Majority of respondents reported that clotrimazole mouth paint $1 \%$ were topical therapeutic is used for the treatment of oral candidiasis. (Pearson chi square value - 27.883; $\mathrm{p}$-value $=0.00<0.05$ which is statistically significant).

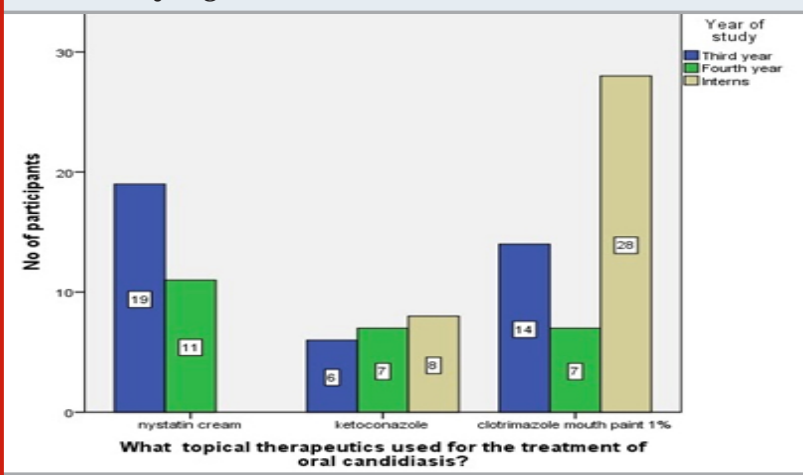

In this study $43 \%$ of the dental students reported that triamcinolone acetonide oro-base $0.1 \%$ were commonly used topical therapeutic in management of aphthous ulcer . Zouboulis et al., reported similar evidence that the combination of topical anesthetics (for example, lidocaine gel) during the day with an oral paste containing triamcinolone acetate also effective in treating aphthous ulcer.(Zouboulis, 2003) In Germany Altenburg et al., also reported similar evidence that the only drugs that have been approved to treat oral aphthous ulcers are corticosteroids ,topical antiseptic /anti-inflammatory agents such as triclosan and diclofenac, and local anesthetics such as lidocaine.(Altenburg et al., 2014)

Graph 32: Bar graph showing comparison of responses based on year of study to the question, "Most common antiseptic mouth rinse prescribed by you in your dental practice?" $\mathrm{X}$ axis represents the questions and $\mathrm{Y}$ axis represents the number of participants .Majority of respondents reported that hexidine mouth rinse were the most common antiseptic mouth rinse prescribed by them in their dental practice(Pearson chi square value - $44.609 ; p$ -value $=0.00<0.05$ which is statistically significant).

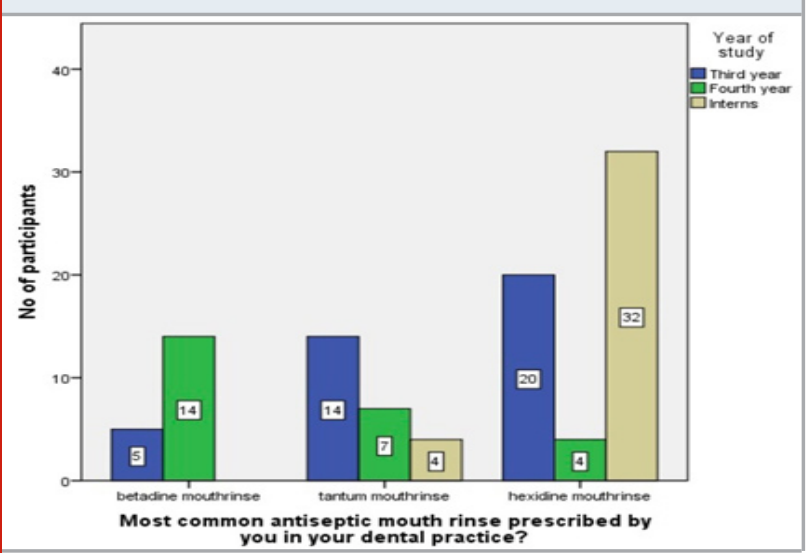

Graph 33: Bar graph showing comparison of responses based on year of study and awareness about topical therapeutics in management of oral mucosal lesion among undergraduate dental students. $X$ axis represents the questions and $\mathrm{Y}$ axis represents the number of participants. Interns were more aware about topical therapeutics in management of oral mucosal lesions. (Pearson chi square value $-60.475 ; \mathrm{p}$-value $=0.00<0.05$ which is statistically significant).

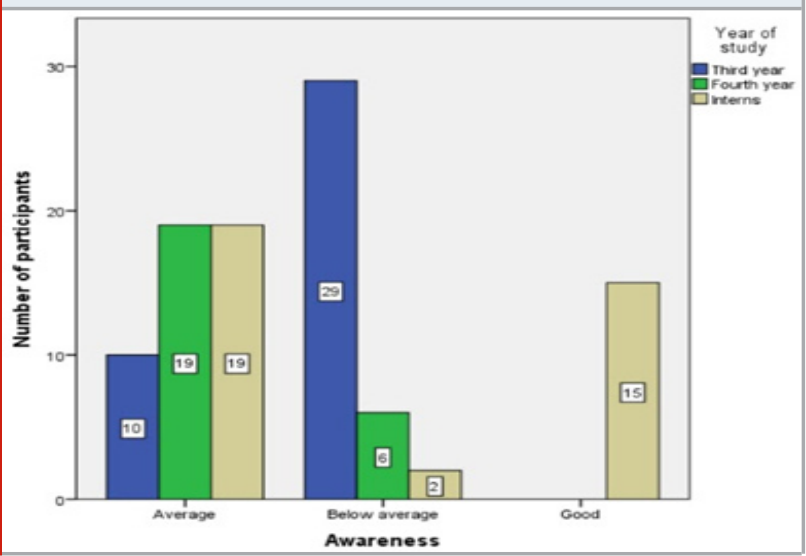

Majority of 49\% respondents reported that clotrimazole mouth paint $1 \%$ were the topical therapeutic used for the treatment of oral candidiasis. Akpan et al reported similar evidence that oral hygiene and topical antifungals were adequate for treating uncomplicated oral candidiasis.(Akpan and Morgan, 2002). This was in accordance with Guru P.E et al which states that $72 \%$ 
of the dental students were aware about the effect of corticosteroids. The study also states that Oral Lichen planus as the most common Oral mucosal lesion ('Oral mucosal lesions', 2015) .A study stated that Oral Lichen planus was the most common oral mucosal lesion .(Daly, 2014) Majority of the dental students also answered that triamcinolone acetate was the treatment of choice for Oral Lichen Planus. (Gupta and Jawanda, 2015) Studies stated that Topical corticosteroids as the first line therapy for mucosal erosive lichen planus. (Omal et al., 2012) Oral Lichen Planus was found to be the most common compared to skin lichen planus.

Majority of dental students were aware that oral lichen planus was the most common oral mucosal lesion for prescribing topical steroids and triamcinolone acetate $0.1 \%$ was one of the common topical steroids in management of oral lichen planus. Kiran et al., reported similar evidence that betamethasone, fluticasone propionate spray, topical mometasone furoate microemulsion, clobetasol propionate in orabase, triamcinolone acetonide (mouthwash, topical, intralesional) had all shown to be effective in the symptomatic management of OLP.(Kiran et al., 2017). Limitations of the study includes dishonest answers in questionnaires by respondents and usage of a single online survey platform in a single university setting. Future study should aim at conducting surveys using multiple online survey platforms to include more participants in different university settings.

\section{CONCLUSION}

Within the limits of the present study, interns (15\%) showed good awareness compared to the other undergraduate dental students followed by final years (19\%) who had average awareness on use of topical therapeutic in management of oral mucosal lesions. Most of the third year dental students (29\%) showed below averaged which emphasise a need on educational programs exclusively on topical therapeutics in management of oral mucosal lesions to be initiated from the beginning of clinical exposure for undergraduate students and educational programs on current updates of topical therapeutics is essential for undergraduates to ensure proper treatment protocol in dental practice.

\section{ACKNOWLEDGEMENTS}

The authors sincerely acknowledge Saveetha Dental College and Hospitals, Saveetha Institute of Medical And Technical Science for granting permission to conduct this questionnaire based study among dental students.

Author Contributions:The first author Nivethitha contributed towards data collection, data analysis and manuscript preparation, the second author, Uma Maheshwari contributed towards the study design, key concept, critical analysis and review of the study.

Conflicts of Interest: There was no conflict of interest as declared by the authors.

\section{REFERENCES}

Ajmal, M. et al. (2018) 'Prevalence and psychological stress in recurrent aphthous stomatitis among female dental students in Saudi Arabia', Medicine and Pharmacy Reports, pp. 216-221. doi: 10.15386/cjmed840.

Akpan, A. and Morgan, R. (2002) 'Oral candidiasis', Postgraduate medical journal, 78(922), pp. 455-459.

Altenburg, A. et al. (2014) 'The treatment of chronic recurrent oral aphthous ulcers', Deutsches Ärzteblatt international, 111(40), pp. 665-673.

Daly, C. (2014) 'Dental note: Topical corticosteroids and oral mucosa', Australian Prescriber, pp. 167-167. doi: 10.18773/austprescr.2014.067.

Eisen, D. et al. (2005) 'Number V Oral lichen planus: clinical features and management', Oral diseases, 11(6), pp. 338-349.

Field, E. A. and Allan, R. B. (2003) 'Oral ulceration aetiopathogenesis, clinical diagnosis and management in the gastrointestinal clinic', Alimentary Pharmacology and Therapeutics, pp. 949-962. doi: 10.1046/j.13652036.2003.01782.x.

González-Moles, M. A. and Scully, C. (2005) 'Vesiculoerosive oral mucosal disease--management with topical corticosteroids: (1) Fundamental principles and specific agents available', Journal of dental research, 84(4), pp. 294-301.

Gupta, S. and Jawanda, M. (2015) 'Oral lichen planus: An update on etiology, pathogenesis, clinical presentation, diagnosis and management', Indian Journal of Dermatology, p. 222. doi: 10.4103/0019-5154.156315. Hearnden, V. et al. (2012) 'New developments and opportunities in oral mucosal drug delivery for local and systemic disease', Advanced Drug Delivery Reviews, pp. 16-28. doi: 10.1016/j.addr.2011.02.008.

Hughes, M. and Ghosh, T. (2015) 'Pharmaceuticals for Oral Mucosal Drug Delivery: Regulatory Considerations', Advances in Delivery Science and Technology, pp. 247-274. doi: 10.1007/978-1-4899-7558-4_11.

Kiran, M. S. et al. (2017) 'Systemic and Topical Steroids in the Management of Oral Mucosal Lesions', Journal of pharmacy \&t bioallied sciences, 9(Suppl 1), pp. S1-S3. Leão, J. C., Gomes, V. B. and Porter, S. (2007) 'Ulcerative lesions of the mouth: an update for the general medical practitioner', Clinics , 62(6), pp. 769-780.

Omal, P. M. et al. (2012) 'Prevalence of oral, skin, and oral and skin lesions of lichen planus in patients visiting a dental school in Southern India', Indian Journal of Dermatology, p. 107. doi: 10.4103/0019-5154.94276. Oral mucosal lesions' (2015) Dental Abstracts, pp. 53-54. doi: 10.1016/j.denabs.2014.05.047.

Parashar, P. (2011) ‘Oral lichen planus', Otolaryngologic 
clinics of North America, 44(1), pp. 89-107, vi.

Patil, S. R. et al. (2018) 'Comparative Study of the Efficacy of Newer Antioxidants Lycopene and Oxitard in the Treatment of Oral Submucous Fibrosis', Pesquisa brasileira em odontopediatria e clínica integrada, 18(1), pp. 1-7.

Patil, S. R. et al. (2018) 'Three-Rooted Mandibular First Molars in a Saudi Arabian Population: A CBCT Study', Pesquisa brasileira em odontopediatria e clínica integrada, 18(1), p. e4133.

Patil, S. R. et al. (2020) 'Assessment of Maximum Bite Force in Oral Submucous Fibrosis Patients: A Preliminary Study', Pesquisa brasileira em odontopediatria e clínica integrada. (Histopathological studies before and after kepacort in oral submucous fibrosis), 20, p. 482.

Subramaniam, N. and Muthukrishnan, A. (2019) 'Oral mucositis and microbial colonization in oral cancer patients undergoing radiotherapy and chemotherapy: A prospective analysis in a tertiary care dental hospital', Journal of investigative and clinical dentistry, 10(4), p. e12454.

Tripathi, K. D. (2013) 'Essentials of Medical Pharmacology'. doi: 10.5005/jp/books/12256.

Vadivel, J. K. et al. (2019) 'Mast cell expression in oral lichen planus: A systematic review', Journal of investigative and clinical dentistry, 10(4), p. e12457.

Yang, S.-F. et al. (2018) 'Changes in prevalence of precancerous oral submucous fibrosis from 1996 to 2013 in Taiwan: A nationwide population-based retrospective study', Journal of the Formosan Medical Association = Taiwan yi zhi, 117(2), pp. 147-152.

Zouboulis, C. C. (2003) 'Adamantiades-Behçet's disease', European Handbook of Dermatological Treatments, pp. 16-26. doi: 10.1007/978-3-662-07131-1_3. 